\title{
Redox-mediated PKA-Rla localisation to the lysosome inhibits myocardial calcium release and robustly reduces myocardial injury
}

Jillian N. Simon ${ }^{1}$, Besarte Vrellaku1, Stefania Monterisi², Sandy Chu ${ }^{1}$, Nadiia Rawlings $^{1}$, Oliver Lomas ${ }^{1}$, Gerard A. Marchal ${ }^{1}$, Dominic Waithe ${ }^{3}$, Parag Gajendragadkar $^{1}$, Raja Jayaram ${ }^{1}$, Keith Channon ${ }^{1}$, Pawel Swietach², Manuela Zaccolo$^{2}$, Phil Eaton ${ }^{4}$, Barbara Casadei ${ }^{1}$

${ }^{1}$ Division of Cardiovascular Medicine, Radcliffe Department of Medicine, University of Oxford, Oxford, UK

2Department of Physiology, Anatomy and Genetics, University of Oxford, Oxford, UK

${ }^{3}$ Wolfson Imaging Centre, Weatherall Institute of Molecular Medicine, University of Oxford, Oxford, UK

${ }^{4}$ Cardiovascular Division, The Rayne Institute, King's College London, London, UK

Background: Kinase oxidation is a critical signalling mechanism through which changes in the intracellular redox state alter cardiac function. In the myocardium, type-1 PKA (PKARla) can be reversibly oxidised, forming interprotein disulfide bonds within the holoenzyme complex. However, the effect of PKARla oxidation on downstream signalling in the heart, particularly under states of oxidative stress, remains unexplored.

Purpose: To determine the direct functional consequences of PKARla oxidation in the heart and investigate their impact on ischaemia/reperfusion $(\mathrm{I} / \mathrm{R})$ injury.

Methods \& Results: Experiments using the AKAR3ev FRET biosensor in murine left ventricular (LV) myocytes and Fluorescence Recovery After Photobleaching (FRAP) of GFP-tagged WT and mutant Rla proteins in Rla-null fibroblasts showed that PKARla oxidation does not increase the kinases's catalytic activity, but enhances its binding to A-kinase anchoring proteins (AKAP; $n=30-39 / N=3, p<0.01$ ). Super-resolution microscopy revealed localisation of oxidised PKARla to lysosomes in WT myocytes, which was completely absent in "redox dead" Cys17Ser PKARIa knock-in mice $(\mathrm{KI}$; panel $A ; \mathrm{n}=38-41 / \mathrm{N}=3, \mathrm{p}<0.01)$ and reduced when AKAP binding was prevented using the RIAD disruptor peptide $(30.6 \pm 5.1 \%$ reduction; $n=35$ $37 / \mathrm{N}=3, \mathrm{p}<0.01)$.

Displacement of PKARla from lysosomes resulted in spontaneous sarcoplasmic reticulum $\mathrm{Ca}^{2+}$ release and dramatic $\mathrm{Ca}^{2+}$ oscillations in $\mathrm{KI}$ LV myocytes (panel $B$ ), which were preventable by ryanodine receptor blockade ( $1 \mathrm{mM}$ tetracaine; $n=14$, $p<0.01$ ), acute depletion of endolysosomal $\mathrm{Ca}^{2+}$ stores (100 nM bafilomycin; $n=7$; $\mathrm{p}<0.01$ ), or lysosomal two-pore channel (TPC) inhibition (5 $\mu \mathrm{M} \mathrm{Ned-19;} n=9$; $\mathrm{p}<0.05)$.

I/R (secondary to cardiopulmonary bypass) was found to induce PKARla oxidation in the myocardium of patients undergoing cardiac surgery (panel $C ; \mathrm{n}=18, \mathrm{p}=0.02$ ). 
Absence of this response in $\mathrm{KI}$ mouse hearts resulted in 2-fold larger infarcts $(p<0.01)$ and a concomitant reduction in LV contractile recovery (final LVDP of $55.9 \pm 8.6$ vs $82.5 \pm 7.1 \mathrm{mmHg}$ in $\mathrm{WT} ; \mathrm{n}=7-8, \mathrm{p}<0.05)$, both which were prevented by addition of Ned-19 at the time of reperfusion (panel $D ; n=4, p<0.01$ ).

Conclusions: Oxidised PKARla acts as a potent inhibitor of intracellular $\mathrm{Ca}^{2+}$ release in the heart through its redox-dependent interaction with the lysosome. In the setting of $\mathrm{I} / \mathrm{R}$, where PKA oxidation is induced, this regulatory mechanism is critical for protecting the heart from injury and offers a novel target for the design of cardioprotective therapeutics.

\section{Funding Acknowledgements:}

British Heart Foundation grants CH/12/3/29609 (to J.N.S., B.C.), RG/16/12/32451(to J.N.S., B.V., B.C.), FS/17/17/32438 (to P.G.), RG/17/6/32944 (M.Z., S.M.), PG/15/5/31110 (to M.Z.), RG/15/9/31534 (to P.S.), and PG/17/44/33064 (to P.E.); Medical Research Council grants MR/P023150/1 (to P.E) and MR/S005382/1 and MRC/BBSRC/EPSRC MR/K01577X/1 (to D.W.); Garfield-Weston Foundation MPS/IVIMS-11/12-4032 (to N.R., B.C.); Wellcome Trust Fellowship 0998981Z/12/Z (to O.L.)

Figure:
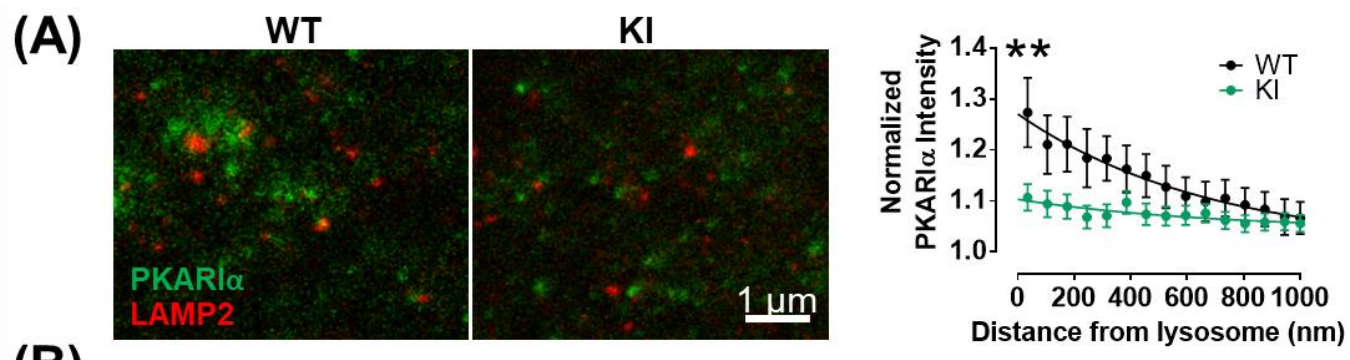

(B)
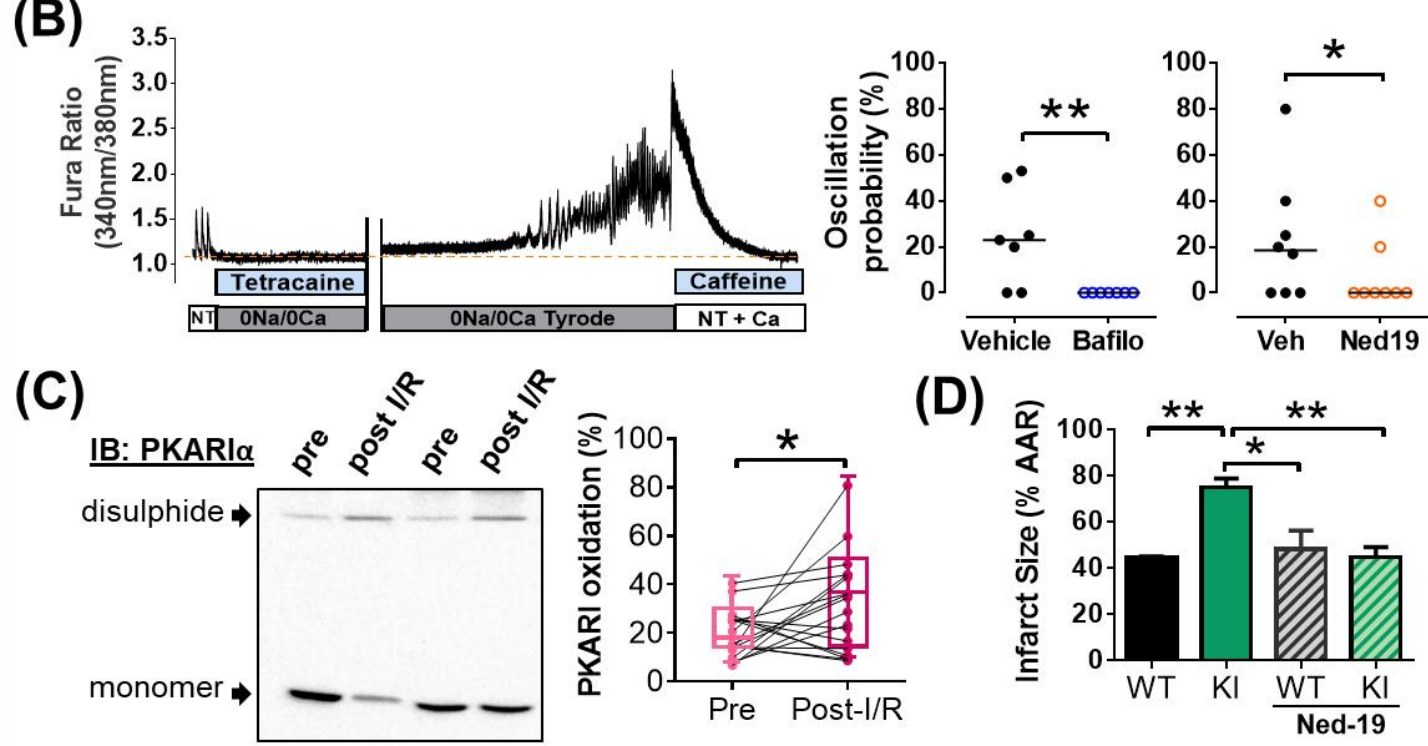

(D)

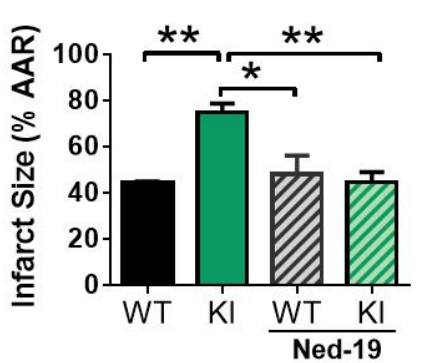

